\title{
Autonomous CaMKII Can Promote either Long-Term Potentiation or Long-Term Depression, Depending on the State of T305/T306 Phosphorylation
}

\author{
Hyun Jae Pi, ${ }^{1,2}$ Nikolai Otmakhov, ${ }^{2}$ David Lemelin, ${ }^{3}$ Paul De Koninck, ${ }^{3}$ and John Lisman ${ }^{2}$ \\ ${ }^{1}$ Quantitative Biology Program, Physics Department, and ${ }^{2}$ Volen Center for Complex Systems, Biology Department, Brandeis University, Waltham, \\ Massachusetts 02454, and ${ }^{3}$ Centre de Recherche Université Laval Robert-Giffard, Quebec, Quebec G1J 2G3, Canada
}

$\mathrm{Ca}^{2+} /$ calmodulin-dependent kinase II (CaMKII) is a key mediator of long-term potentiation (LTP). Whereas acute intracellular injection of catalytically active CaMKII fragments saturates LTP (Lledo et al., 1995), an autonomously active form (T286D) of CaMKII holoenzyme expressed in transgenic mice did not saturate potentiation (Mayford et al., 1995). To better understand the role of the holoenzyme in the control of synaptic strength, we transfected hippocampal neurons with constructs encoding forms of CaMKII mimicking different phosphorylation states. Surprisingly, T286D not only failed to potentiate synaptic strength, but produced synaptic depression through an long-term depression (LTD)-like process. T305/T306 phosphorylation was critical for this depression because overexpression of the pseudophosphorylated form (T286D/T305D/T306D) caused depression that occluded LTD, and overexpression of an autonomous form in which T305/T306 could not be phosphorylated (T286D/T305A/T306A) prevented LTD (instead producing potentiation). Therefore, autonomous CaMKII can lead to either LTP or LTD, depending on the phosphorylation state of the control point, T305/T306.

\section{Introduction}

A single neuron in the brain has thousands of synapses, each of which can be modified bidirectionally in response to different patterns of neuronal activity (Heynen et al., 1996; Enoki et al., 2009). The activity-dependent processes that change synaptic strength are called long-term potentiation (LTP) and long-term depression (LTD) (Malenka and Bear, 2004).

$\mathrm{Ca}^{2+} /$ calmodulin-dependent kinase II (CaMKII) has been implicated as a central molecule in the induction of LTP (Lisman et al., 2002). It is known that CaMKII can be switched by $\mathrm{Ca}^{2+}$ dependent autophosphorylation of T286 to a state in which the enzyme is active even in the absence of $\mathrm{Ca}^{2+}$ (autonomous) (Miller and Kennedy, 1986). Induction of LTP produces an increase in CaMKII activity as a result of autophosphorylation of this site (Fukunaga et al., 1993; Fukunaga et al., 1995). Both pharmacological and genetic experiments show that this activation is necessary for LTP induction (Malinow et al., 1989; Otmakhov et al., 1997; Giese et al., 1998). Other experiments show that active CaMKII can mimic LTP (Pettit et al., 1994; Lledo et al., 1995; Hayashi et al., 2000; Poncer et al., 2002; Jourdain et al., 2003). Notably, acute intracellular injection of active CaMKII fragments (CaMKII:1-290) saturates synaptic potentiation and occludes induction of LTP by synaptic stimulation (Lledo et

Received Jan. 8, 2010; revised March 23, 2010; accepted March 26, 2010.

This work was supported by National Institutes of Health (NIH) Grant R01 NS027337-19 and National Institute of Neurological Disorders and Stroke-NIH Grant R01 NS050944, and the Canadian Institutes of Health Research (CIHR). P.D.K. is a CIHR Young Investigator Awardee. We thank all members of the Lisman laboratory for helpful comments on this manuscript and Dr. K. J. Kang (Brandeis University) for useful discussions.

Correspondence should be addressed to John Lisman at the above address. E-mail: lisman@brandeis.edu.

DOI:10.1523/JNEUROSCI.0133-10.2010

Copyright $\odot 2010$ the authors $\quad 0270-6474 / 10 / 308704-06 \$ 15.00 / 0$ al., 1995). Similarly, overexpression of constitutively active CaMKII fragment produces LTP (Pettit et al., 1994; Hayashi et al., 2000; Poncer et al., 2002). Together, these results strongly suggest that CaMKII can activate the biochemistry that underlies LTP induction.

There are, however, results that do not fit with this picture. Based on the experiments described above, it would be expected that synapses in a transgenic animal containing an autonomous form of CaMKII holoenzyme (T286D) should exhibit saturated potentiation. However, this prediction failed, as indicated by the fact that normal LTP still could be induced by high-frequency stimulation (Mayford et al., 1995).

We have sought to better understand the role of autonomous CaMKII holoenzyme in synaptic modification. In previous work, the effect of T286D on synaptic strength could not be directly compared with control cells. Here, we used a cellular transfection system that allows such comparison. Surprisingly, we found that the T286D CaMKII caused synaptic depression and that this depression occluded LTD. Based on previous findings that T305/ T306 phosphorylation of CaMKII is involved in metaplasticity, we hypothesized that this depression occurs because T305/T306 becomes phosphorylated. Indeed, when phosphorylation at these sites was prevented, autonomous CaMKII did not produce LTD; instead, synaptic strength was increased to a saturated LTP state. When the phosphorylation is mimicked, a synaptic depression that occluded LTD was observed. Thus, the autonomous form of CaMKII can regulate synaptic strength bidirectionally, depending upon its phosphorylation state at T305/T306. The identification of different phosphorylation states that can saturate LTP or LTD provides new molecular tools for manipulating these processes. 
A

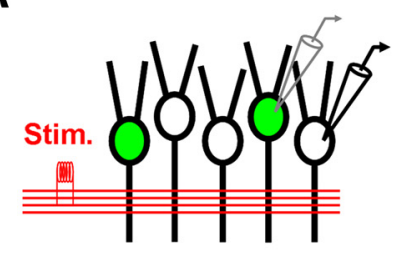

B
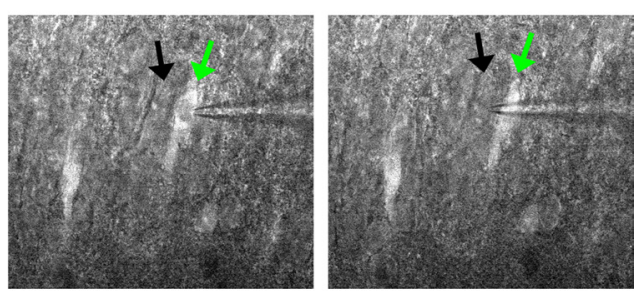

D
C

nontransfected transfected
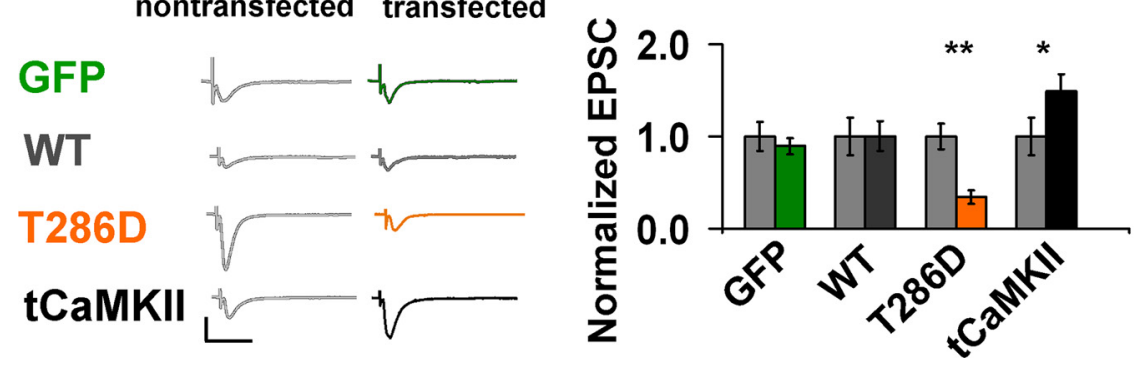

Figure 1. T286D decreases AMPAR EPSC, whereas tCaMKII increases it. $A$, Cell pair recordings were performed from transfected (green) and nontransfected (white) neurons. B, An example of cell pair recordings. Left, Recording from transfected neuron (green arrow). Right, Recording from nontransfected neuron (black arrow). $C$, The representative traces of AMPAR EPSC from nontransfected and transfected neurons. Calibration: 50 pA, 50 ms. D, T286D decreases AMPAR EPSC, whereas tCaMKII increases it (see Results for details). GFP, Nontransfected $=51.8 \pm 8.10 \mathrm{pA}$, transfected $=46.3 \pm 4.65 \mathrm{pA}, n=15, p=0.33$; WT, nontransfected $=58.7 \pm 12.02$, transfected $=58.9 \pm 9.43 \mathrm{pA}, n=7, p=0.98 ;$ T286D, nontransfected $=117.1 \pm 16.16 \mathrm{pA}$, transfected $=39.8 \pm 8.59 \mathrm{pA}, n=14,{ }^{* *} p<0.01$; tCaMKII, transfected $=112.8 \pm 14.36 \mathrm{pA}$, nontransfected $=75.9 \pm 15.47$ $\mathrm{pA}, n=12,{ }^{*} p<0.05$. For T286D experiments, the stimulation strength was raised to avoid noisy or undetectable small responses due to the depression.

\section{Materials and Methods}

Organotypic slice culture. Hippocampal slice cultures were prepared as described by Stoppini et al. (1991) and Otmakhov et al. (2004).

DNA constructs and transfection. The green fluorescent protein (GFP)tagged full-length $\alpha$ CaMKII constructs (WT, T286D) used in this study were described by Hudmon et al. (2005). The GFP contained the A207K mutation to ensure its monomeric state (mGFP). The T286D/T305A/ T306A $\alpha$ CaMKII mutant was made by restriction digest and ligation of mGFP T286D $\alpha$ CaMKII vector with T305A/T306A- $\alpha$ CaMKII insert using $\mathrm{XmnI}$ and MfeI. The same approach was used to generate the T286D/ T305D/T306D mutation. All constructs were verified by sequence analysis. The truncated form (tCaMKII-GFP) was a gift from Y. Hayashi (RIKEN Brain Science Institute, Wako City, Japan). After 10-17 d in vitro, single-cell electroporation (SCE) was used for targeted transfection as described by Haas et al. (2001) and Rathenberg et al. (2003). Using SCE, slice cultures were transfected with plasmids containing mGFP $\alpha$ CaMKII variant (wildtype, tCaMKII, T286D, T286D/T305A/T306A, and T286D/T305D/T306D).

Electrophysiology. Slices were continuously superfused $(1.8 \mathrm{ml} / \mathrm{min})$ with artificial CSF (ACSF) containing the following (in mM): $124 \mathrm{NaCl}$, $2.5 \mathrm{KCl}, 4 \mathrm{CaCl}_{2}, 4 \mathrm{MgCl}_{2}, 1.25, \mathrm{NaH}_{2} \mathrm{PO}_{4}, 26 \mathrm{NaHCO}_{3}, 0.05$ picrotoxin, and $0-1 \mu \mathrm{M} 2$-chloroadenosine, balanced with $95 \% \mathrm{O}_{2}$ and $5 \% \mathrm{CO}_{2}, \mathrm{pH}$ 7.4. Whole-cell recordings were performed at room temperature (22$24^{\circ} \mathrm{C}$ ). The pipette solution contained (in $\mathrm{mm}$ ) 120 Csmethanesulfonate, $20 \mathrm{CsCl}, 10$ HEPES, 4 MgATP, $0.3 \mathrm{Na}_{3} \mathrm{GTP}, 0.2$ EGTA, and $5 \mathrm{Na}_{2}$-phosphocreatine, pH 7.3; osmolarity, $320 \mathrm{mOsm}$. EPSCs were evoked by stimulating the Schaffer-collateral pathway via a stimulation electrode and measured in voltage-clamp mode at $-65 \mathrm{mV}$. For cell pair recordings, two neurons (one transfected and one nontransfected) within $\sim 30 \mu \mathrm{m}$ were selected. The test stimulation was delivered every $6 \mathrm{~s}$ with $0.2 \mathrm{~ms}$ duration, and the intensity $(100-500 \mu \mathrm{A})$ was adjusted to induce 50-100 pA EPSC. After adjusting stimulation strength in one neuron, sequential recording was performed in the other neuron with the same intensity and location of stimulation. The peak amplitudes of EPSC were averaged with 30-50 sweeps. Series and input resistance were monitored throughout each experiment, and recordings for which series resis- tance varied by $>20 \%$ were rejected. For experiments with drug preincubation, $100 \mu \mathrm{M}$ DL-2-amino-5-phosphono-valeric acid (APV) and $50 \mu \mathrm{M}$ FK-506 were added immediately after transfection for each experiment, and after 22-26 $\mathrm{h}$, the recordings were performed in ACSF without drugs. Some analysis was performed blind to experimental conditions. Because the results were similar, they were combined with data obtained in nonblinded experiments.

LTP and LTD induction. As reported informally by many laboratories, LTP and LTD are not induced reliably in cultured slices under standard conditions, possibly because cultured slices develop burst-like responses to synaptic stimulation when inhibition is totally blocked. Not blocking inhibition at all is problematic because it becomes difficult to induce LTP (Wigström and Gustafsson, 1983). LTP was induced by a pairing protocol using 200 pulses at $2 \mathrm{~Hz}$ at $0 \mathrm{mV}$. LTD was induced by pairing 300 pulses at $1 \mathrm{~Hz}$ at $-45 \mathrm{mV}$. The response to test pulses was measured at $-70 \mathrm{mV}$. We found that partial block of inhibition (1-2 $\mu \mathrm{M}$ picrotoxin) prevents burst-like responses and allows reliable LTP and LTD induction (58 successes/62 attempts; $\sim 93 \%$; induction was done $>1$ $\mathrm{h}$ after cutting off CA3). At $-70 \mathrm{mV}$ holding voltage, the IPSC is inward, but contributes only $\sim 20 \%$ to the evoked current. Thus, the contamination by the IPSC is minor and equally present in transfected and nontransfected neurons. The LTP and LTD induced under these conditions are NMDAR dependent (supplemental Fig. S5, available at www.jneurosci.org as supplemental material).

Statistics. Two-tailed paired Student's $t$ tests were performed. All results are reported as mean \pm SEM.

\section{Results}

To understand the role of different phosphorylation states of CaMKII holoenzyme in synaptic plasticity, we used single-cell electroporation to express different forms of $\alpha$ CaMKII tagged with monomeric green fluorescent protein (GFP- $\alpha$ CaMKII) in CA1 pyramidal neurons in cultured hippocampal slices. The level of $\alpha$ CaMKII expression in the soma, $24 \mathrm{~h}$ after transfection, was estimated by immunostaining with antibodies specific for $\alpha$ CaMKII (supplemental Fig. S1 $A$, available at www.jneurosci. org as supplemental material). This staining was approximately threefold higher than the endogenous level in nearby neurons (supplemental Fig. S1 B, available at www.jneurosci.org as supplemental material).

Approximately $24 \mathrm{~h}$ after transfection of CaMKII variants, synaptic responses were compared between transfected neurons and nearby nontransfected neurons (Fig. 1) (see Materials and Methods). In the neurons expressing a truncated, constitutively active form of CaMKII (tCaMKII; 1-290), the amplitude of AMPAR EPSC was larger than that of neighboring nontransfected neurons, consistent with previous reports (Hayashi et al., 2000; Poncer et al., 2002) (Fig. 1C,D). In contrast, overexpression of either wild-type CaMKII or GFP had no effect (Fig. 1C,D).

We next transfected neurons with the complete holoenzyme containing the mutation, T286D. This form is pseudophosphorylated on the autoregulatory site, keeping the enzyme in an active state, independent of $\mathrm{Ca}^{2+} / \mathrm{CaM}$ binding (autonomous) (Lou and Schulman, 1989). We expected that it would enhance 


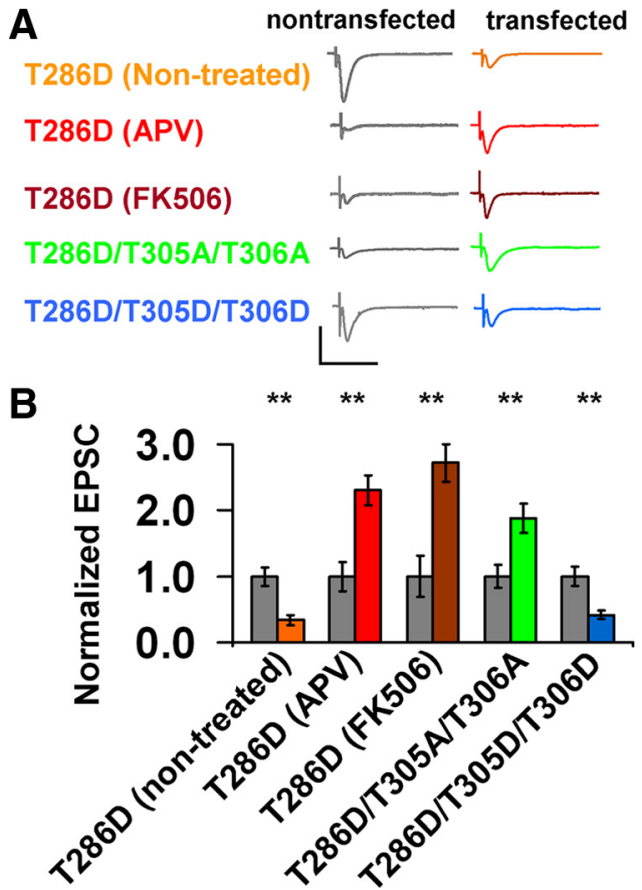

Figure 2. T286D-dependent depression is reversed by an NMDAR antagonist (APV), calcineurin inhibitor (FK-506), or additional mutation at T305A/T306A. Gray, Control condition; orange, T286D in nontreated culture medium; red, T286D in culture medium containing APV; brown, T286D in culture medium containing FK-506; green, triple mutant (T286D/T305A/ T306A). $\boldsymbol{A}$, The representative EPSC traces. Calibration: $50 \mathrm{~ms}, 50$ pA. $\boldsymbol{B}$, T286D-dependent depression is reversed by an NMDAR antagonist, calcineurin inhibitor, or additional mutation at T305A/T306A. T286D, Nontransfected $=117.1 \pm 16.16 \mathrm{pA}$, transfected $=39.8 \pm 8.59 \mathrm{pA}, n=$ $14,{ }^{* *} p<0.01 ;$ T286D (APV), transfected $=66.1 \pm 6.57 \mathrm{pA}$, nontransfected $=28.7 \pm 6.41 \mathrm{pA}$, $n=8,{ }^{* *} p<0.01 ; \mathrm{T} 286 \mathrm{D}$ (FK506), transfected $=100.7 \pm 10.44 \mathrm{pA}$, nontransfected $=37.1 \pm$ $11.57 \mathrm{pA}, n=9,{ }^{*} p<0.05 ; \mathrm{T} 286 \mathrm{D} / \mathrm{T} 305 \mathrm{~A} / \mathrm{T} 306 \mathrm{~A}$, transfected $=80.1 \pm 9.39 \mathrm{pA}$, nontransfected $=$ $42.4 \pm 7.51 \mathrm{pA}, n=18,{ }^{*} p<0.05 ; \mathrm{T} 286 \mathrm{D} / \mathrm{T} 305 \mathrm{D} / \mathrm{T} 306 \mathrm{D}$, transfected $=31.3 \pm 4.95 \mathrm{pA}$, nontransfected $=74.5 \pm 10.99 \mathrm{pA}, n=22,{ }^{* *} p<0.01$.

synaptic transmission in a manner similar to the truncated active form discussed above. To our surprise, the T286D form produced a substantial decrease in the AMPAR EPSC (Fig. 1C,D).

In cultured slices, NMDAR activation by spontaneous neural activity can induce synaptic plasticity during the $\geq 1 \mathrm{~d}$ period between transfection and testing (Hayashi et al., 2000; Zhu et al., 2002). This raises the possibility that the depression caused by T286D CaMKII is due to an activity-dependent process similar to LTD. To test this hypothesis, we examined whether the T286Ddependent depression was blocked by APV, an agent that blocks a common form of LTD (Dudek and Bear, 1992). Slices were incubated in culture medium containing $100 \mu \mathrm{M}$ APV (NMDAR blocker), beginning immediately after transfection and continuing to just before the time of testing. Supportive of the above hypothesis, APV blocked the depression and resulted in potentiation (Fig. 2).

NMDAR-dependent LTD has been shown to require the activity of the $\mathrm{Ca}^{2+}$-dependent phosphatase, PP2B (calcineurin) (Mulkey et al., 1994; Zeng et al., 2001). Consistent with this, we found that incubating the slices in the calcineurin inhibitor, FK$506(50 \mu \mathrm{M})$, blocked the depression produced by T286D CaMKII and resulted in potentiation (Fig. 2). We similarly tried to test the role of PP1 (Mulkey et al., 1993, 1994; Nicholls et al., 2008) but were unsuccessful because inhibitors of this phosphatase (calyculin A: $0.2 \mu \mathrm{M}, 0.4 \mu \mathrm{M}$, or $1 \mu \mathrm{M}$ ) blocked expression of transfected CaMKII through an unknown mechanism. Together, these results indicate that the weakening process produced by T286D CaMKII shares substantial similarities with LTD. The depression is not due to NMDAR-dependent action of the test pulses (supplemental Fig. S2, available at www.jneurosci.org as supplemental material). Furthermore, consistent with a change in synaptic strength, we did not find evidence that T286D CaMKII produces significant changes in the number of axons approaching transfected cells (supplemental Fig. S3, available at www.jneurosci.org as supplemental material).

What might determine whether potentiation or depression of synaptic transmission is mediated by T286D CaMKII? The holoenzyme has multiple phosphorylation sites on each subunit. Among these, the T305/T306 site is within the calmodulinbinding domain; the phosphorylation of this site prevents activation by $\mathrm{Ca}^{2+} /$ calmodulin (Lou and Schulman, 1989). Under in vitro conditions of low $\mathrm{Ca}^{2+}$, wild-type CaMKII that has been phosphorylated at T286 becomes autophosphorylated at T305/ T306 (Lou and Schulman, 1989). Phosphorylation of this site, which makes it harder to produce LTP induction and easier to induce LTD (Elgersma et al., 2002; Zhang et al., 2005), is implicated in metaplasticity. We hypothesized that the activity in the T286D form might autophosphorylate T305/T306 under low $\mathrm{Ca}^{2+}$ conditions, leading to a state of CaMKII that promotes LTD.

If the phosphorylation at T305/T306 is causal for depression, the prevention of phosphorylation at these sites should block depression, whereas the phosphomimetic at these sites should produce depression. Consistent with these predictions, the triple mutation T286D/T305A/T306A, which cannot be phosphorylated at T305/T306, prevented the depression and led instead to potentiation of synaptic transmission (Fig. 2). Furthermore, expression of the phosphomimetic form, T286D/T305D/T306D, caused depression (Fig. 2). These results thus support the hypothesis that phosphorylation of T305/T306 causes the synaptic depression. The results also imply that the T286D mutation itself supports NMDAR-independent potentiation but that additional phosphorylation at T305/T306 sets in motion a process that causes NMDAR-dependent depression (Fig. 2).

If this potentiation and depression are forms of LTP and LTD, the depression caused by T286D and T286D/T305D/T306D mutants should occlude further LTD, and the potentiation produced by T286D/T305A/T306A CaMKII should occlude further LTP. To test this prediction, LTP and LTD were induced with pairing protocols applied to transfected and neighboring nontransfected neurons. As found in other laboratories, under standard conditions, the reliability of LTP induction is poor in cultured slices. However, with addition of low concentration of picrotoxin, we were able to induce LTP and LTD in $>90 \%$ of attempts (see Materials and Methods, LTP and LTD induction). Under these conditions, we tested the types of plasticity that could be induced in the depressed state caused by expression of the T286D form. Nontransfected control neurons showed LTD that produced an $\sim 50 \%$ decrease in the AMPAR EPSC (Fig. $3 B$ ). However, LTD induction failed to depress AMPAR EPSC in neurons expressing T286D-CaMKII, indicating that T286D CaMKII-dependent depression occluded LTD (Fig. 3B). LTP in T286D CaMKIItransfected neurons was comparable to that in nontransfected neurons (Fig. 3A).

Next, we compared plasticity in neurons transfected with T286D/T305A/T306A CaMKII and neighboring nontransfected neurons. Whereas nontransfected neurons showed robust LTP, neurons expressing T286D/T305A/T306A CaMKII had almost no LTP. This indicates that T286D/T305A/T306A CaMKII- 

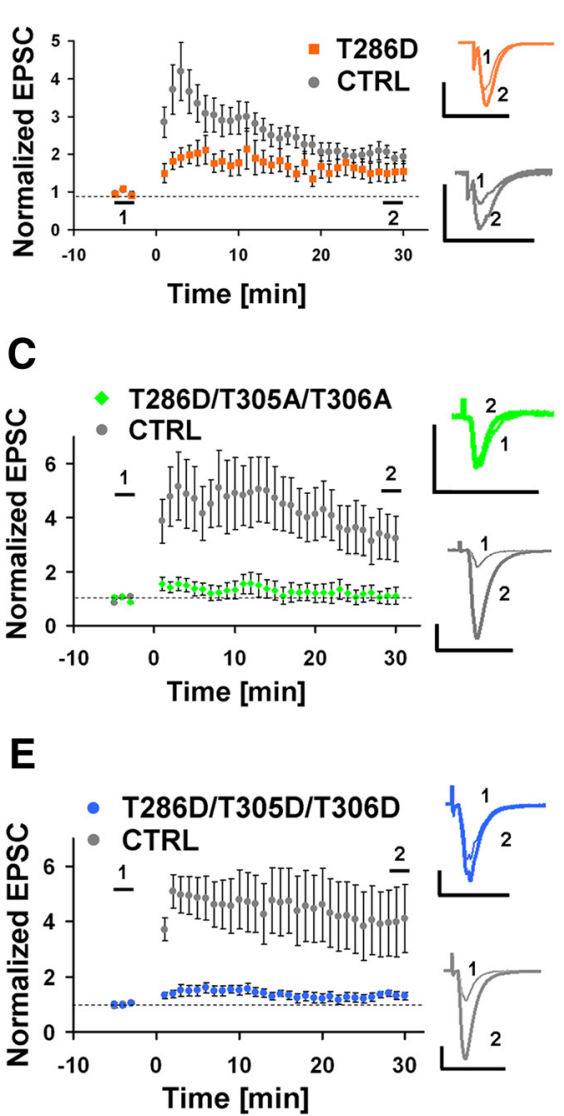

B

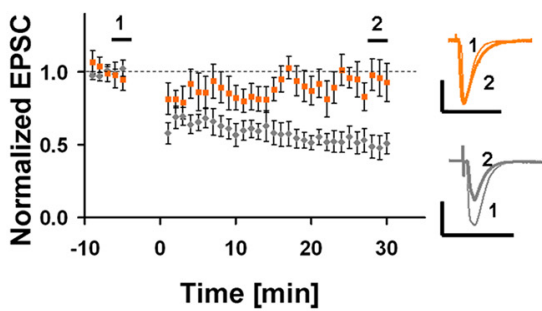

D

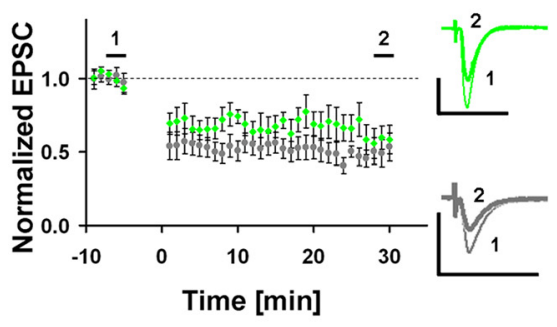

$\mathbf{F}$

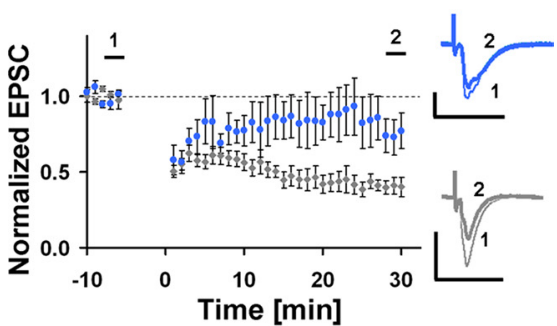

Figure 3. T286D occludes LTD, T286D/T305A/T306A occludes LTP, and T286D/T305D/T306D occludes both LTP and LTD. Normalized EPSC amplitudes plotted against time from transfected (orange, T286D; green, T286D/T305A/T306A; and blue, T286D/ T305D/T306D) and neighboring nontransfected (gray) neurons during baseline and after LTP/LTD. 1, Baseline; 2, 30 min after LTP/LTD. The level of LTP/LTD is measured at 2. Calibration: $50 \mathrm{pA}, 50 \mathrm{~ms}$. A, LTP is intact in T286D. The level of LTP was as follows: T286D $=1.53 \pm 0.267, n=11 ; \mathrm{CTRL}=1.97 \pm 0.165, n=10,{ }^{*} p<0.05 . B$, LTD is occluded in T286D. The level of LTD was as follows: T286D $=0.96 \pm 0.122, n=8 ;$ CTRL $=0.49 \pm 0.078, n=8,{ }^{* *} p<0.01$. C, LTP is occluded in T286D/T305A/T306A. The level of LTP: T286D/T305A/T306A $=1.08 \pm 0.272, n=8 ;$ CTRL $=3.30 \pm 0.870, n=8,{ }^{* *} p<0.01$. D, LTD is intact in T286D/T305A/T306A. The level of LTD was as follows: T286D/T305A/T306A $=0.58 \pm 0.093, n=8 ;$; CTL $=0.51 \pm 0.093, n=$ $8, p=0.06 . E$, LTP is occluded in T286D/T305D/T306D. The level of LTP was as follows: T286D/T305D/T306D $=1.34 \pm 0.135, n=$ $10 ; \mathrm{CTRL}=4.01 \pm 1.227, n=10,{ }^{* *} p<0.01 . \boldsymbol{F}, \mathrm{LTD}$ is occluded in T286D/T305D/T306D. The level of LTD was as follows: $\mathrm{T} 286 \mathrm{D} / \mathrm{T} 305 \mathrm{D} / \mathrm{T306D}=0.75 \pm 0.114, n=10 ; \mathrm{CTRL}=0.40 \pm 0.054, n=10,{ }^{* *} p<0.01$.

dependent potentiation occluded LTP (Fig. 3C). Neurons expressing this triple mutant showed intact LTD comparable to nontransfected neighboring neurons (Fig. 3D).

Last, we examined plasticity in neurons expressing T286D/ T305D/T306D CaMKII. Nontransfected nearby neurons in the same slices had robust LTP and LTD (Fig. $3 E, F$ ). However, this triple phosphomimetic mutant occluded further LTD (Fig. $3 F$ ). Notably, this mutant also blocked LTP (Fig. 3E). The latter result is to be expected because pseudophosphorylation at T305/T306 prevents the binding of $\mathrm{Ca}^{2+} /$ calmodulin to the enzyme, which prevents not only its $\mathrm{Ca}^{2+} /$ calmodulin-dependent activation, but also its activity-dependent translocation to the postsynaptic density (Shen and Meyer, 1999; Elgersma et al., 2002; Hudmon et al., 2005). Together, these results indicate that the potentiation and depression produced by different phosphorylation states of CaMKII are affecting the LTP and LTD processes, respectively.

\section{Discussion}

We have made the first systematic study of how different phosphorylation states of CaMKII affect synaptic strength. Previous work showed that CaMKII made active by truncation of the regulatory region produces an increase in synaptic strength that occludes LTP (Pettit et al., 1994; Lledo et al., 1995). We have confirmed that such strengthening occurs and expected that any active form of CaMKII would strengthen synapses. However we found, surprisingly, that holoenzyme made active by pseudophosphorylation at T286 (T286D) produces a decrease in synaptic strength. Furthermore, this decrease appears to be a form of LTD because, like LTD, it is dependent on NMDAR function and on phosphatase activity; moreover, the weakening occludes LTD.

The finding that the T286D form does not produce potentiation helps to explain surprising findings on the properties of plasticity in a transgenic mouse having overexpression of T286D (Mayford et al., 1995). In these experiments, the effect of CaMKII on synaptic strength could not be measured because there was no control pathway. However, from the fact that large LTP could be induced, it was clear that large potentiation had not occurred. This is consistent with our finding that, in synapses that have undergone LTD-like weakening by T286D, large LTP can be induced.

A second major finding regards the role of T305/T306 in determining whether LTP or LTD will occur. The importance of these sites is demonstrated by the finding that forms pseudophosphorylated at these sites (T286D/T305D/T306D) result in LTD (Fig. 2). In contrast, preventing phosphorylation at these sites (T286D/T305A/T306A) produces LTP. Similarly, if the T305/T306 sites are deleted (as in the catalytically active truncated form; tCaMKII), then LTD is prevented. It has been shown in vitro that T305/ T306 gets phosphorylated after T286 site is phosphorylated (Lou and Schulman, 1989; Jama et al., 2009). Given that CaMKII can become phosphorylated at T305/T306 in living cells (Elgersma et al., 2002), the weakening caused by overexpression of the T286D form probably is likely to be secondary to T305/T306 phosphorylation.

In considering how T286D produces weakening, three points first need to be made. First, previous works (Zhu et al., 2000, 2002; Barria and Malinow, 2005) on cultured slices have indicated that the slices are endogenously active and can produce firing patterns that affect plasticity (specifically, the strengthening and weakening produced by small GTPases, wild-type Ras, and Rap, respectively, are NMDAR dependent). The firing patterns vary diversely both in frequency and amplitude (Zhu et al., 2000; Barria and Malinow, 2005). Second, knock-in mice with CaMKII pseudophosphorylated at T305/T306 have altered metaplasticity: only the strongest activity patterns can produce LTP; other patterns produce LTD (Elgersma et al., 2002; Zhang et al., 2005). Thirdly, in our experiments with CaMKII variants, the weakening and strengthening have a different dependence on 
NMDARs. When induced synaptically, both LTP and LTD in CA1 are NMDAR dependent. However, in our experiments, potentiation is produced by the T286D form in the absence of NMDAR function. This indicates that, under these conditions, kinase activity directly targets the biochemical processes that underlie strengthening. In contrast, autonomously active CaMKII (T286D) cannot produce weakening when NMDARs are blocked. This implies that the LTD biochemical machinery is not directly affected; rather, the weakening relies on NMDARdependent LTD stimulated by spontaneous activity.

With this as a framework, we interpret our results as follows. The ongoing spontaneous LTP-like and LTD-like events increase kinase and phosphatase activity, respectively. Part of the increase in kinase activity will be due to the activation of wild-type kinase. There is also basal kinase activity produced by the autonomous transfected CaMKII mutants. Whether weakening or strengthening occur will depend on the balance of all of these activities and the state of metaplasticity. As suggested by the involvement of T305/T306 in metaplasticity (Zhang et al., 2005) and Angelman syndrome (Weeber et al., 2003; van Woerden et al., 2007), we assume that the cross-over point in the Bienenstock-CooperMunro (BCM) curve (Bienenstock et al., 1982) that relates LTP and LTD to firing frequency will be shifted to the right if T305/ T306 is phosphorylated (supplemental Fig. S4, available at www. jneurosci.org as supplemental material). Under these conditions, most firing patterns will induce LTD, and this will shift the net effect of all firing patterns toward LTD (supplemental Fig. S4, available at www.jneurosci.org as supplemental material). However, the metaplasticity shift to the right is not total; there are still very strong firing patterns that can induce LTP, and this explains why LTP can be induced experimentally in the T286D form by strong synaptic stimulation. If T305/T306 phosphorylation does not occur, the shift does not occur, and now many activity patterns produce LTP, shifting the net balance in favor of LTP (Fig. 2). Finally, if the effects of activity are blocked by APV, now the only process that affects plasticity is the direct enzymatic effect of autonomous CaMKII; under these conditions, synapses are strengthened (Fig. 2).

In a mouse model of Angelman's syndrome, there is an increased level of phosphorylation at T286 and T305/T306 (Weeber et al., 2003). In these mice, the BCM curve is shifted to the left, as also occurs in T286D transgenic mice (Mayford et al., 1995). Moreover, in both types of mice, LTP is not saturated. Our results provide the first direct demonstration that if T286 and T305/ T306 become phosphorylated, a weakening process is stimulated and LTP is therefore not saturated. These results thus help to explain why mutations that prevent T305/T306 phosphorylation rescue the behavioral deficits in Angelman's syndrome (van Woerden et al., 2007) and underscore the importance of understanding why this phosphorylation occurs aberrantly in the disease.

The molecular mechanisms by which T305/T306 affects metaplasticity also remain to be determined. Phosphorylation at T305/T306 reduces CaMKII binding to PSD (Elgersma et al., 2002), which could affect the strengthening process. A recent study shows that CaMKII interaction with $\alpha$-actinin, an actinbundling protein, is interrupted by T305/T306 phosphorylation (Robison et al., 2005).

Our results provide new methods for genetically altering the strength and plasticity of synapses. The T286D form weakens synapses and prevents further LTD. The T286D/T305A/T306A form saturates potentiation and blocks LTP but permits LTD. The T286D/T305D/T306D form weakens synapses and blocks both LTP and LTD, putting the synapse in a locked state. These tools may be useful for testing the role of LTP/LTD in learning and memory.

\section{References}

Barria A, Malinow R (2005) NMDA receptor subunit composition controls synaptic plasticity by regulating binding to CaMKII. Neuron 48:289-301.

Bienenstock EL, Cooper LN, Munro PW (1982) Theory for the development of neuron selectivity: orientation specificity and binocular interaction in visual cortex. J Neurosci 2:32-48.

Dudek SM, Bear MF (1992) Homosynaptic long-term depression in area CA1 of hippocampus and effects of N-methyl-D-aspartate receptor blockade. Proc Natl Acad Sci U S A 89:4363-4367.

Elgersma Y, Fedorov NB, Ikonen S, Choi ES, Elgersma M, Carvalho OM, Giese KP, Silva AJ (2002) Inhibitory autophosphorylation of CaMKII controls PSD association, plasticity, and learning. Neuron 36:493-505.

Enoki R, Hu YL, Hamilton D, Fine A (2009) Expression of long-term plasticity at individual synapses in hippocampus is graded, bidirectional, and mainly presynaptic: optical quantal analysis. Neuron 62:242-253.

Fukunaga K, Stoppini L, Miyamoto E, Muller D (1993) Long-term potentiation is associated with an increased activity of $\mathrm{Ca} 2+/$ calmodulindependent protein kinase II. J Biol Chem 268:7863-7867.

Fukunaga K, Muller D, Miyamoto E (1995) Increased phosphorylation of $\mathrm{Ca} 2+/$ calmodulin-dependent protein kinase II and its endogenous substrates in the induction of long-term potentiation. J Biol Chem 270:6119-6124.

Giese KP, Fedorov NB, Filipkowski RK, Silva AJ (1998) Autophosphorylation at Thr286 of the alpha calcium-calmodulin kinase II in LTP and learning. Science 279:870-873.

Haas K, Sin WC, Javaherian A, Li Z, Cline HT (2001) Single-cell electroporation for gene transfer in vivo. Neuron 29:583-591.

Hayashi Y, Shi SH, Esteban JA, Piccini A, Poncer JC, Malinow R (2000) Driving AMPA receptors into synapses by LTP and CaMKII: requirement for GluR1 and PDZ domain interaction. Science 287:2262-2267.

Heynen AJ, Abraham WC, Bear MF (1996) Bidirectional modification of CA1 synapses in the adult hippocampus in vivo. Nature 381:163-166.

Hudmon A, Lebel E, Roy H, Sik A, Schulman H, Waxham MN, De Koninck P (2005) A mechanism for $\mathrm{Ca}^{2+} /$ calmodulin-dependent protein kinase II clustering at synaptic and nonsynaptic sites based on self-association. J Neurosci 25:6971-6983.

Jama AM, Fenton J, Robertson SD, Török K (2009) Time-dependent autoinactivation of phospho-Thr286-alphaCa2 +/calmodulin-dependent protein kinase II. J Biol Chem 284:28146-28155.

Jourdain P, Fukunaga K, Muller D (2003) Calcium/calmodulin-dependent protein kinase II contributes to activity-dependent filopodia growth and spine formation. J Neurosci 23:10645-10649.

Lisman J, Schulman H, Cline H (2002) The molecular basis of CaMKII function in synaptic and behavioural memory. Nat Rev Neurosci 3: 175-190.

Lledo PM, Hjelmstad GO, Mukherji S, Soderling TR, Malenka RC, Nicoll RA (1995) Calcium/calmodulin-dependent kinase II and long-term potentiation enhance synaptic transmission by the same mechanism. Proc Natl Acad Sci U S A 92:11175-11179.

Lou LL, Schulman H (1989) Distinct autophosphorylation sites sequentially produce autonomy and inhibition of the multifunctional $\mathrm{Ca}^{2+} /$ calmodulindependent protein kinase. J Neurosci 9:2020-2032.

Malenka RC, Bear MF (2004) LTP and LTD: an embarrassment of riches. Neuron 44:5-21.

Malinow R, Schulman H, Tsien RW (1989) Inhibition of postsynaptic PKC or CaMKII blocks induction but not expression of LTP. Science 245:862-866.

Mayford M, Wang J, Kandel ER, O’Dell TJ (1995) CaMKII regulates the frequency-response function of hippocampal synapses for the production of both LTD and LTP. Cell 81:891-904.

Miller SG, Kennedy MB (1986) Regulation of brain type II Ca2+/ calmodulin-dependent protein kinase by autophosphorylation: a $\mathrm{Ca} 2+-$ triggered molecular switch. Cell 44:861-870.

Mulkey RM, Herron CE, Malenka RC (1993) An essential role for protein phosphatases in hippocampal long-term depression. Science 261: 1051-1055.

Mulkey RM, Endo S, Shenolikar S, Malenka RC (1994) Involvement of a 
calcineurin/inhibitor-1 phosphatase cascade in hippocampal long-term depression. Nature 369:486-488.

Nicholls RE, Alarcon JM, Malleret G, Carroll RC, Grody M, Vronskaya S, Kandel ER (2008) Transgenic mice lacking NMDAR-dependent LTD exhibit deficits in behavioral flexibility. Neuron 58:104-117.

Otmakhov N, Griffith LC, Lisman JE (1997) Postsynaptic inhibitors of calcium/calmodulin-dependent protein kinase type II block induction but not maintenance of pairing-induced long-term potentiation. J Neurosci 17:5357-5365.

Otmakhov N, Tao-Cheng JH, Carpenter S, Asrican B, Dosemeci A, Reese TS, Lisman J (2004) Persistent accumulation of calcium/calmodulindependent protein kinase II in dendritic spines after induction of NMDA receptor-dependent chemical long-term potentiation. J Neurosci 24:9324-9331.

Pettit DL, Perlman S, Malinow R (1994) Potentiated transmission and prevention of further LTP by increased CaMKII activity in postsynaptic hippocampal slice neurons. Science 266:1881-1885.

Poncer JC, Esteban JA, Malinow R (2002) Multiple mechanisms for the potentiation of AMPA receptor-mediated transmission by alpha- $\mathrm{Ca}^{2+}$ / calmodulin-dependent protein kinase II. J Neurosci 22:4406-4411.

Rathenberg J, Nevian T, Witzemann V (2003) High-efficiency transfection of individual neurons using modified electrophysiology techniques. J Neurosci Methods 126:91-98.

Robison AJ, Bartlett RK, Bass MA, Colbran RJ (2005) Differential modulation of $\mathrm{Ca} 2+/$ calmodulin-dependent protein kinase II activity by regulated interactions with $\mathrm{N}$-methyl-D-aspartate receptor NR2B subunits and alpha-actinin. J Biol Chem 280:39316-39323.

Shen K, Meyer T (1999) Dynamic control of CaMKII translocation and localization in hippocampal neurons by NMDA receptor stimulation. Science 284:162-166.

Stoppini L, Buchs PA, Muller D (1991) A simple method for organotypic cultures of nervous tissue. J Neurosci Methods 37:173-182.

van Woerden GM, Harris KD, Hojjati MR, Gustin RM, Qiu S, de Avila Freire R, Jiang YH, Elgersma Y, Weeber EJ (2007) Rescue of neurological deficits in a mouse model for Angelman syndrome by reduction of alphaCaMKII inhibitory phosphorylation. Nat Neurosci 10:280-282.

Weeber EJ, Jiang YH, Elgersma Y, Varga AW, Carrasquillo Y, Brown SE, Christian JM, Mirnikjoo B, Silva A, Beaudet AL, Sweatt JD (2003) Derangements of hippocampal calcium/calmodulin-dependent protein kinase II in a mouse model for Angelman mental retardation syndrome. J Neurosci 23:2634-2644.

Wigström H, Gustafsson B (1983) Facilitated induction of hippocampal long-lasting potentiation during blockade of inhibition. Nature 301:603-604.

Zeng H, Chattarji S, Barbarosie M, Rondi-Reig L, Philpot BD, Miyakawa T, Bear MF, Tonegawa S (2001) Forebrain-specific calcineurin knockout selectively impairs bidirectional synaptic plasticity and working/episodiclike memory. Cell 107:617-629.

Zhang L, Kirschstein T, Sommersberg B, Merkens M, Manahan-Vaughan D, Elgersma Y, Beck H (2005) Hippocampal synaptic metaplasticity requires inhibitory autophosphorylation of $\mathrm{Ca}^{2+} /$ calmodulin-dependent kinase II. J Neurosci 25:7697-7707.

Zhu JJ, Esteban JA, Hayashi Y, Malinow R (2000) Postnatal synaptic potentiation: delivery of GluR4-containing AMPA receptors by spontaneous activity. Nat Neurosci 3:1098-1106.

Zhu JJ, Qin Y, Zhao M, Van Aelst L, Malinow R (2002) Ras and Rap control AMPA receptor trafficking during synaptic plasticity. Cell 110:443-455. 